\title{
Control of place-cell activity in an open field
}

\author{
SAM A. DEADWYLER, CHARLES R. BREESE, and ROBERT E. HAMPSON \\ Bowman Gray School of Medicine, Wake Forest University, Winston-Salem, North Carolina
}

\begin{abstract}
The nature of place-cell firing in the hippocampus is discussed with relevance to control of discharge characteristics in a "cue-sterile" open-field environment. Although it can be demonstrated that cell firing in this context is controlled by spatial influences, it is also shown that firing within the place field and the location of the place field can be altered by nonspatial influences as well. Within-field firing in this environment is controlled to a large degree by the trajectory of the animal's movements. Analyses involving forward or backward time shifts in firing, adjusted for variation in speed of movement, trajectory convergence, and number of spikes, produced no indication that complex cell firing reflects either memory for, or anticipated arrival in, locations other than where the cell fired originally. Nor is the location of the place field strictly dependent upon the spatial features of the apparatus, since it is shown that place-cell firing can be changed readily by altering the significance or relevance of a particular location. Place-cell plasticity in this context suggests nonspatial control over where place cells fire. These results are considered in terms of hippocampal cell coding of specific types of spatial information. Considerable discussion is devoted to whether coding of place is shifted in the same cell, or whether there is emergence of place firing by a different cell (detected by the same electrode), as an explanation for this apparent plasticity. Although no resolution is provided, the implications of these findings for a strict spatial mapping theory of the hippocampus are addressed and other interpretations are explored.
\end{abstract}

Cells in the central nervous system (CNS) that can code for spatial locations within the environment present several problems for neurobiological approaches to understanding how the brain works. Given that all the necessary sensory information for such a calculation is present (in some form or other) in the CNS, the issue of whether this information can be accessed by a particular structure or cell with such specificity as to enable "place-specific" firing is currently under intense investigation. Apparent placespecific firing by complex spike cells in the hippocampus, as first reported by O'Keefe and Dostrovsky (1971), suggests that this is one structure in which spatial coding might take place. Besides being repeatedly verified in several laboratories (see Eichenbaum \& Cohen, 1988), the discovery of such "place cells" has produced a major theory of hippocampal function (O'Keefe \& Nadel, 1978). Recently, however representation of spatial versus nonspatial information by complex spike cells has been an issue of considerable interest among investigators in the field. This volume represents some interesting contrasting views of place-specific firing by hippocampal complex spike cells. Utilizing different assessment techniques in order to understand the nature of place-cell firing, it has become readily apparent from the disparity of results

This investigation was supported (in part) by Research Scientist Award DA00119 from the ADAMHA awarding institute NIDA, as well as NIDA Grants DA03502 and DA04441 to S.A.D. The efforts of Sonia Stitcher in helping to prepare the manuscript are greatly appreciated. Correspondence may be addressed to Sam A. Deadwyler, Department of Physiology and Pharmacology, Bowman Gray School of Medicine, Wake Forest University, Winston-Salem, NC 27103. in the field that an absence of agreement as to the conceptual and neurobiological bases of the phenomenon exists.

Our investigations of place-cell activity have centered on two major questions: (1) What controls the within-field firing of place cells? (2) Are place fields fixed and rigidly controlled by the spatial features of the environment, or can they be altered by nonspatial influences that control behavior? These two topics will be considered in the following discussion.

\section{Directional Specificity of Place-Cell Firing}

Directional firing of hippocampal place cells has been shown within several different contexts (McNaughton, Barnes, \& O'Keefe, 1983; Muller, Kubie, \& Ranck, 1987). Place cells in the hippocampus do not code absolute directional features since hippocampal cell fields shift location to maintain position relative to prominent spatial cues if those cues are rotated (Muller \& Kubie, 1987; O'Keefe, 1979; O'Keefe \& Conway, 1978; Olton, Walker, \& Gage, 1978). This is in contrast with cells in the postsubiculum (Ranck, 1984; Taube, Muller, \& Ranck, 1988). Determination of direction-specific firing of place cells is somewhat confounded since movement in a particular direction also constitutes moving into or out of a place field. Since place fields tend to be distributed in different physical (spatial) locations within the environment, absolute directional control is difficult to assess. We recently showed (Breese, Hampson, \& Deadwyler, 1987) that relative directional firing differentiates placecell discharges in terms of turning biases in the following manner: (1) cells with symmetric turning correlates 
fired maximally when the animal was moving straight ahead within the field and fired less as the animal deviated from a straight trajectory, (2) other cells displayed maximal firing when the animal turned right (right-skew cells) or left (left-skew cells) within the field, and (3) still other cells had no obvious directional biases. Although these preliminary analyses could be influenced by other factors, such as head turning (Bostock, Taube, \& Muller, 1988), they are in agreement with recordings from cells in the parietal cortex of the rat which apparently code directional sensitivity in a similar manner (McNaughton, 1989).

There are other aspects of in-field firing of place cells that suggest relative rather than absolute directional sensitivity. Movement trajectories associated with cell firing in the field can be divided into three different classes: those associated with entering the field (i.e., the animal entered the field and stopped), leaving the field (the animal left the field after stopping within its boundaries), or passing through the field (the animal did not stop while in the field). These calculations revealed that $81 \%$ of all entering trajectories ( $n=13$ animals) were associated with significantly $(p<.01)$ increased firing, whereas fewer trajectories were associated with leaving $(51 \%)$ or passing through the field (41\%). A similar analysis by McNaughton, Barnes, and O'Keefe (1983) of firingassociated trajectories in an eight-arm maze revealed a firing bias for inward trajectories in that task as well.

On an open platform, therefore, firing of place cells is influenced to a large extent by relative direction and traversal patterns of the animal. We have confirmed a report by Muller and Kubie (1987) showing that strategically placed barriers that interrupt established movement trajectories reduce or suppress place-cell firing even though the animal can enter the field via another direction or route (Breese, Hampson, \& Deadwyler, 1988, 1989). Foster, Castro, and McNaughton (1988) eliminated place-specific discharges by adapting animals to physical immobilization and passively moving them through the field. This suggests that self-initiated movements within the field are crucial for place-specific firing. Muller and Kubie considered movement a major controlling factor in their "kinematic" explanation of place-cell firing. A recent study of hippocampal involvement in objectplace associations in primates by Parkinson, Murray, and Mishkin (1988) points out the necessity of considering the significance of purposive movement toward a particular location within a defined spatial context as a potentially powerful controlling aspect of place-cell firing.

\section{Firing Within the Field:}

\section{A Spatial or Temporal Correlate?}

Given that in-field firing of place cells is closely associated with the animal's traversal pattern, it is important to determine the nature of this movement-related correlate. Is the cell discharge signaling (1) the momentary or immediate spatial location that the animal occupies as it moves through the field (O'Keefe \& Nadel, 1978;
Zipser, 1985), (2) the step-by-step movement patterns that propel the animal from one location in the field to the next (Kubie, Fox, \& Muller, 1984), or (3) the anticipated arrival in a spatial location that the animal will subsequently occupy while traversing through the field (Muller \& Kubie, 1986)? The latter issue was addressed by Muller and Kubie (1986) using a time-shift analysis of the array of spikes in relation to the array of coordinates of spike locations within the field. The two arrays were shifted until the field with the highest spike density (i.e., maximal field constriction) was obtained. This was quite a perceptive manipulation since, presumably, a time shift that produced the smallest field size would "predict" the location of the animal at a time that was different from actual spike occurrence. Optimal field constriction was shown to be associated with a positive (forward) time shift of approximately $160 \mathrm{msec}$ following the time of actual spike occurrence. The time-shift analysis suggests that place-cell firing represents "anticipated" arrival in a different location, possibly the next physical location along a given trajectory (Muller \& Kubie, 1986).

Time-shift analyses can potentially reveal very important aspects of place-cell firing; however, interpretation of the meaning of field changes produced by such analyses must be approached with caution. We were very interested in the validity of this measure and its potential importance; therefore, several time-shift analyses were performed examining different measures of spike distribution within the field, including spike density, field area, and distance between spikes (Breese et al., 1989). No evidence of a significant time shift was obtained with any of the above measures. However, three confounds in the time-shift calculation must be ruled out before a timeshifted constriction of field size can be considered a valid reflection of temporal coding by place cells.

First, the array of spikes that is time-shifted cannot vary due to alterations in the velocity of the animal's movements through the field. If this occurs, there will be an artificial "pile up" of spikes (constriction of the field) at time-shifted locations where there are a changes in velocity. Second, the number of spikes in the analysis must remain constant even if they are time-shifted out of original field boundaries. A loss of spikes because of shifting out of the limits of the original field would constrict the field through the selective loss of dispersed spikes. Third, movement trajectories associated with the spike array cannot converge from different directions. Trajectory convergence directly constricts the field because the timeshifted spike locations will also converge and will be maximally condensed when shifted toward the point of intersection of the trajectories. The resulting time-shifted constriction of the field size reflects a necessary reduction in spike dispersion because of the convergence of movement trajectories to a single locus. Constriction of field size by the above factors has different interpretations than a "prediction" of arrival in a particular spatial location (Muller \& Kubie, 1986). If movement velocity within the 
field did not vary, and if all spikes were counted and trajectories within the field did not converge to a single locus (i.e., were parallel), then, with the above contaminating influences ruled out, we found no significant effect of the time-shift analysis. This raises an interesting question. If place cells did fire in anticipation of arrival in a particular location in the environment, would constriction of field size actually be a measure of this tendency? There is no a priori reason to expect the field to constrict versus (merely) to shift location uniformly along the set of trajectories associated with the animal's movements through the field.

\section{Plasticity of Place-Cell Firing}

The coding of place by the selective discharge of hippocampal complex spike cells has been an underlying assumption since the original observation of place-specific firing (O'Keefe \& Dostrovsky, 1971). This assumption requires that such firings inherently "represent" some spatial feature(s) of the environment. Evidence of a spike code indicative of this function is nonexistent. It has been suggested that each place cell reflects a component of a larger, more complete representation of the spatial features of the environment (O'Keefe \& Speakman, 1987; Zipser, 1985). This interpretation is supported by the selective effects of hippocampal lesions on spatial memory and spatial navigation (Morris, 1983; Olton, Becker, \& Handelmann, 1979). However, even casual observation reveals that animals sustaining hippocampal lesions are not rendered incapable of successfully negotiating spatial barriers and other obstacles that block a path to a goal (i.e., they do not appear to be spatially "blind"), as might be expected if place-cell firing represented critical spatial aspects of the animal's world. A much more specific hypothesis regarding hippocampal involvement in spatial memory is necessary to bridge the gap between the proposed impairment produced by hippocampal damage and the type of spatial information contained in the firing pattern of complex spike cells.

\section{Place-Cell Plasticity: Two Cells or One?}

The known firing characteristics of hippocampal complex spike cells therefore do not explain fully the processing of spatial information. How rigid are the spatial firing tendencies of hippocampal place cells? If place cells encode critical aspects of the spatial features of the environment, either relational (Eichenbaum \& Cohen, 1988) or absolute (O'Keefe \& Speakman, 1987), the firing patterns elicited by those spatial features should be relatively stable. If it can be shown that complex spike cell firing is plastic and changeable within a fixed spatial context, the significance of the type of spatial information encoded by these cells would require alternatives to a strict spatial mapping interpretation.

Central to the issue of whether place cells have variable spatial firing tendencies or have more than one spatial correlate is the possibility that two different place cells with different place fields may be recorded by the same electrode (McNaughton, O'Keefe, \& Barnes, 1983). Recently, it has been proposed that multiple electrodes may be necessary to identify different complex spike cells recorded from a single electrode. A single electrode recording the activity of one cell with a place field might also record another cell firing close by the same electrode. If the waveforms of the two cells were similar, it might not be possible to differentiate them correctly as two cells with individual fields rather than one cell with two different fields. Since place cells appear to have very specific firing correlates, it is important to be able to distinguish between these two possibilities when observing a change in the location of the place field. The stereotrode technique proposed by McNaughton, O'Keefe, and Barnes uses spike-amplitude ratios compared across different electrodes to verify recordings from the same cell. A mismatch in this measure (i.e., different amplitude ratios on the same two electrodes) is assumed to represent the contribution of a different, previously "silent" cell (Thompson \& Best, 1988). The technique illustrates the potential problem in which cells that lie close enough together to be recorded from a single $20-\mu$ wire (microwire) electrode may be indistinguishable as two cells by waveform shape and amplitude criteria, and may be mistakenly assumed to have more than one spatial correlate. O'Keefe \& Speakman (1987), using this technique, showed that different place fields recorded simultaneously from two microwire electrodes reflected activity from different cells.

Two major changes in hippocampal chronic-unit recording have made it necessary to use stereotrode recording procedures: (1) the desire to record from the same cell over long time periods (days and weeks; see Best \& Thompson, 1989) and (2) the desire to record simultaneously from several different identified cells from a single-wire electrode. The relatively large, flexible microwire electrodes that are semipermanently implanted and allowed to "drift" next to cells as they are slowly lowered into the hippocampus (Kubie, 1984) have proven effective for these purposes. Microwire electrodes, however, because of their large surface area, their inability to be precisely positioned, and their tendency to drift next to other cells over days, make stereotrode spike detection and categorization a necessary means of maintaining cell identity (McNaughton, O'Keefe, \& Barnes, 1983). Although other single-electrode chronic recording techniques (e.g., higher impedance "etched" electrodes mounted in microdrives) also have inherent difficulties (i.e., lower probability of long-term recording from the same cell), the contention that stereotrode recording is required for separating multiple spikes detected by any single electrode is not necessarily true and rests on the premise that sufficient isolation of spikes from more than one cell cannot be achieved by physical means.

The arguments in favor of microwire recording are valid, and the quality control achievable by spikeamplitude and time comparisons are notable accomplishments (McNaughton, O'Keefe, \& Barnes, 1983). However, it is a bit extreme to suppose that identical cells 

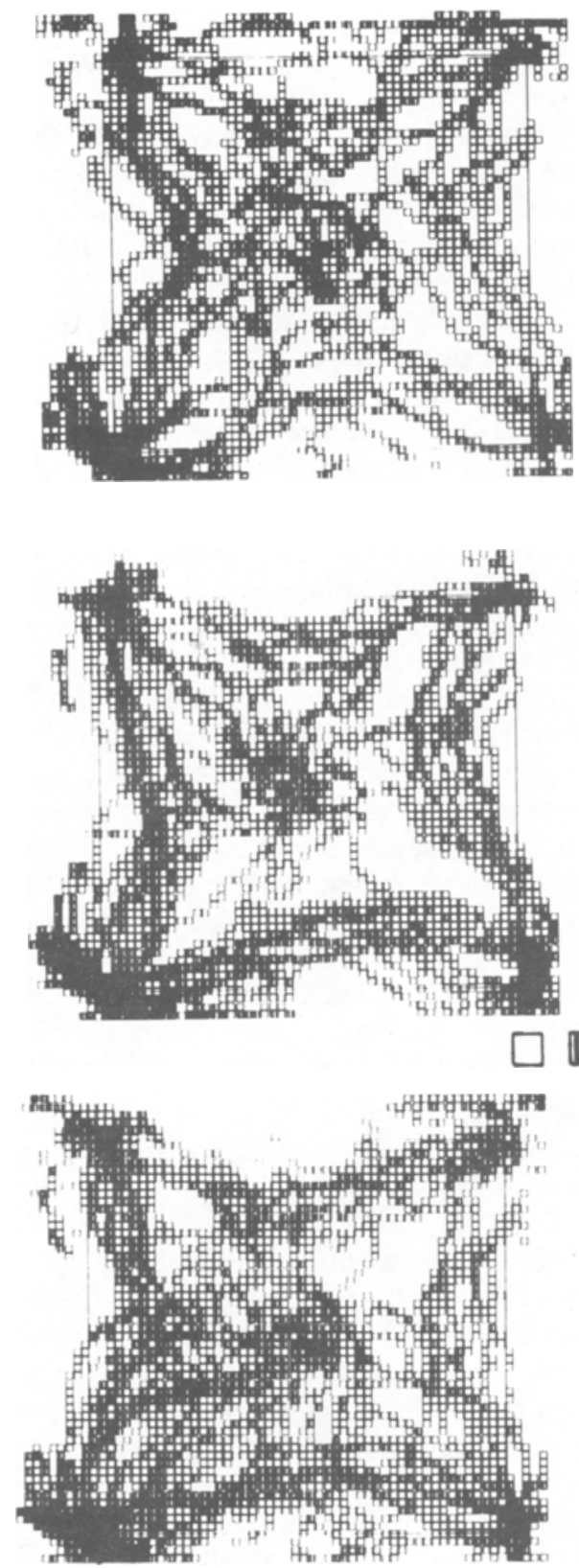

TRAVERSAL MAP
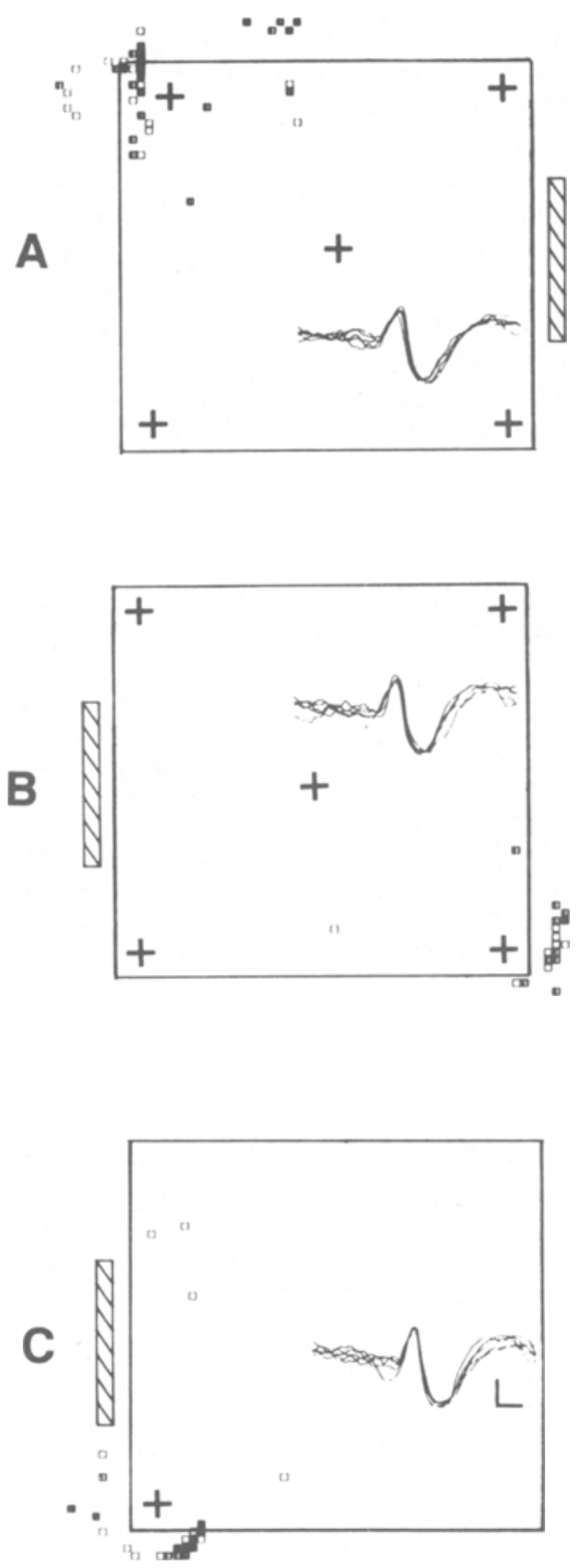

FIRING MAP

Figure 1. Demonstration of plasticity of place-cell firing. (A) Place-cell firing map (right) and traversal map (time in location, left) obtained during a single, 8-min recording session when all five locations on the platform were baited $(+)$. The outline of a square is the area of the platform. The striped bar at the right represents the placement of a large white polarization cue card. The inset shows superimposed (five) traces of the extracellular spike waveform of a complex spilke cell taken during this session. Pixels located of the platform resulted from cable overhang as the rat leaned over the edge of the platform. (B) Firing map showing rotation of the place field from the upper left corner of the diggram to the lower right corner that occurred as the card was rotated to the opposite wall of the enclosure. Cell firing was maintained at the same relative position to the polarizing cue card but was shifted in relation to static room and piatform cues, illustrating effective spatial control over the place field. The inset shows superimposed traces from the same cell (8min session) recorded 15 min anter the session shown in Panel A. (C) The restricted baiting procedure produced a shift in the rotated place field to the corner opposite that shown in Panel B. The animal remained on the platform and the cue card remained in the same position as the session in Panel B. The recording session was initiated $8 \mathrm{~min}$ after restriction of baiting to the lower left corner of diagram $(+)$. Superimposed traces of action potentials from the same cells are shown in the inset. Note that the traversal (time-in-location) maps show little variation across all three recording sessions (left columns of Panels A-C). The gray scale shown in the middle of the figure (left to right) designates $<3.0,4.0-6.0,7.0-9.0$, and $>9.0$ spikes/sec for open, striped, stippled, and filled squares, respectively, for firing maps; and $<0.08,0.09-0.16,0.17-0.24$, and $>0.24 \mathrm{sec}$ for open, striped, stippled, and filled squares, respectively, for traversal maps. Calibration for action-potential waveforms: $2.0 \mathrm{msec}$ and $200 \mu \mathrm{V}$. 
with identical waveforms and amplitudes would be sampled on every occasion with all types of single electrode, or that the presence of other cells would be undetectable during the course of recording with a single etched-tip electrode mounted in a microdrive. In prior studies of place-cell firing, we employed single-electrode recording methods and strict criteria for identifying and maintaining activity from single complex spike cells. Cellular identity was provided by antidromic activation, collision tests, spontaneous firing rate, and different behavioral correlates in two different behavioral tasks (Christian \& Deadwyler, 1986).

\section{Nonspatial Control of Place-Cell Firing}

The issue of whether complex spike cells can be shown to change their firing location when spatial attributes remain unaltered hinges upon the obvious (but nontrivial) assumption that the recordings are from the same cell. We have recently shown that apparent shifts in complex spike cell spatial firing can occur in a "cue-sterile" openfield environment that contains a platform, curtains, and a single, large white polarizing stimulus card on one wall (Breese et al., 1988, 1989). If animals are trained to find water randomly delivered to each cup located in the corners and the middle of the platform, one or more place fields "emerge" when recording from identified hippocampal output neurons (Christian \& Deadwyler, 1986). The place field(s) are usually associated with one, or sometimes two, of the five cup locations (Figure 1A). The manipulation required to change the location of the place field in this task is the restriction of water delivery to only one of the five previously baited locations (Figure IC). After a field has been determined in one location, two prominent changes in place-cell firing are noted when the place field is shifted by the above manipulation: (1) the field is moved to the vicinity of restricted water delivery on the platform, and (2) the cell ceases to fire in the original field location(s). The characteristics of the shifted fields are also altered, including the size and number of firing locations in comparison with the original field (Breese et al., 1988).

Given the current state of knowledge, there are two explanations of this phenomenon that have important implications for the significance of place-cell firing and what it represents. The first is that the electrode detects initiation of firing of a new cell that had not been firing previously (see above). A shift in the place-field location resulting from the initiation of firing in a second (previously silent) cell would not be surprising. However, an as yet undisclosed feature of place-cell activity would be the simultaneous cessation of firing in the originally detected cell. Since the spatial features of the environment are unaltered, it is unclear why a new place cell would initiate firing and why the originally recorded cell would stop firing, even though the traversal patterns and number of visits to both field locations were similar before and after the shift (Figure 1C). An alternative explanation is that the field shift is a consequence of the same cell firing in a new location. This would implicate water delivery as the controlling attribute of place coding by the cell. Several experimental procedures have confirmed that the latter manipulation is the critical feature for shifting the cell (Breese et al., 1989).

Considering the first of the above alternatives, two questions are of relevance: (1) Why is this particular manipulation (water delivery) so effective in initiating firing of a cell that was previously silent (Thompson \& Best, 1988)? (2) Why is the "triggering" of new place-cell firing coincidental with the cessation of firing in the former location? Since these changes occur within identical spatial contexts, strict coding of the spatial features of the environment becomes difficult to accept without modification. The latter concept requires that different place cells have different "mapping features" controlled by nonspatial influences.

The second alternative, that the place field does shift and that the location of firing is dependent upon the significance of a particular spatial location, is more in line with current views of place coding by the hippocampus in the primate literature (Parkinson et al., 1988; Rupniak \& Gaffan, 1987). However, this view also presents problems for a strict spatial mapping theory. First, if "significance" of a location can alter the spatial correlate of a place cell, other cells in the hippocampus that also code spatial location must be shifted accordingly (and precisely) in order to maintain the same relative mapping features after the shift. This is presumably what happens when fields are shifted by rotating the prominent spatial cues within the environment (Figure 1B). However, if the spatial correlates of other place cells are shifted to the same (i.e., restricted baiting) location as the recorded cell (Figure 1C), then the outcome is more serious, since the resulting map would be either inaccurate or destroyed. We have observed only a few instances in which a change in the place correlate did not occur after this manipulation ( 7 of 47 cells). This suggests that most place cells would change their spatial correlate to the same (restricted baiting) location, thereby eliminating the mapping capacity across the population of cells. Whether all fields are changed to the same (restricted baiting) location as the recorded cell, or place fields are rotated to maintain relationally similar spatial locations, is currently under investigation.

A clear case of a failure to show place-specific firing associated with the location of reward is the report of O'Keefe and Speakman (1987). These investigators examined place-cell firing on an elevated "plus" maze while manipulating the location of controlled spatial cues in a room with background or fixed spatial cues. Place fields were localized to the maze arms consistent with the positions of the movable cues in the room. Even though the task required correct movements to a goal (arm) where reward was present, place-cell firing occurred no more frequently on the goal arm than on the other three arms. Of 55 units investigated, 26 showed place fields restricted to one or two arms (although a few showed multiple 
fields). In view of the shifted fields on the platform, goalarm-associated firing would have been expected to be higher in this task, since by definition that particular location acquired enough associative significance to drive the behavior. Records from a single animal showed firing of presumably eight different cells distributed throughout the maze in all four arms, which constituted compelling evidence for the spatial map interpretation of firing in the task (O'Keefe \& Speakman, 1987). However, 65\% of the 26 place cells reported were recorded from only 2 animals, with the remaining 10 cells distributed across an additional 6 animals. Nevertheless, these findings are difficult to reconcile with situations in which goal-oriented complex spike cell firing has been shown by ourselves and others (Eichenbaum \& Cohen, 1988; Eichenbaum, Kuperstein, Fagan, \& Nagode, 1987; Wiener \& Eichenbaum, 1988). It is possible that the plus maze places a large emphasis (through training) on strategies leading to the solution of a unique spatial problem on each trial. Pathways in elevated mazes are fixed and would appear to require little navigational skill in calculating trajectories to particular spatial locations. Under these conditions, the significance of the goal arm may not be as critical as the location from which the correct turn must be made. The lack of preferred firing in any location in the plus maze may reflect the acquired significance of only a few available movement trajectories leading to the perceived goal locations.

\section{Is Location the Only Determinant of Place-Cell Firing?}

The above discussion suggests that several variables, in addition to the presence of the animal in a particular spatial location, control the firing of complex spike cells. Although spatial cues control the firing of place cells under some circumstances, this tendency is easily overcome in circumstances that do not involve attending to spatial features (cf. Berger, Rinaldi, Weisz, \& Thompson, 1983; Best \& Thompson, 1984; Foster, Christian, Hampson, Campbell, \& Deadwyler, 1987). In recent years, evidence for behavioral influences on spatial firing of complex spike cells has been accumulating. Eichenbaum et al. (1987) showed that complex spike cell firing in an odordiscrimination task was most frequent in locations where operant responses were executed or in places associated with movements toward the food dispenser. A direct comparison of place versus reward-associated (cue-sampling) firing revealed that complex spike cells that exhibited place correlates in a spatial navigation task also fired in relation to cue presentation in an odor-discrimination task (Wiener \& Eichenbaum, 1988). Thus, a given complex spike cell can have two separate firing locations within the same environment, each distinctly correlated with the particular contingencies of the behavioral task operative at the time. Similarly, Olton and his co-workers have provided evidence that complex spike cells respond during the delay period of a working-memory task (Wible et al., 1986), a result similar to that of Watanabe and Niki
(1985). In this instance, complex spike cell firing was also dissociable from the strict spatial aspects of the task. Given the above reports, it is only a minor inductive leap to assume that complex spike cells can change their spatial firing correlates within the same task in response to task demands or situations that have altered the significance of particular locations for those behaviors.

Whether the selective baiting influence on place-cell firing in an open platform reflects a specific feature of the sterility of this form of spatial task, or whether the lack of goal-oriented firing of place cells in the plus maze (O'Keefe \& Speakman, 1987) reflects the necessity for specifically coded spatial information to "reconstruct" paths to the goal in that task, it is clear that the two results constitute opposite extremes of a continuum of complex spike cell spatial firing correlates. As mentioned above, both results are difficult to incorporate into a single theory of place-specific firing of complex spike cells. The existence of such precise coding of spatial locations by complex spike cells that emerges spontaneously in several different types of experimental situations (Best \& Ranck, 1982) makes it difficult to reconcile the presence of nonspatial control within similar spatial contexts. Clearly, the above issues indicate that complex spike cells require further investigation with regard to their functional roles in behavior and in the processing of spatial and nonspatial information by the hippocampus.

\section{REFERENCES}

Berger, T. W., Rinaldi, P. C., Weisz, D. J., \& Thompson, R. F. (1983). Single-unit analysis of different hippocampal cell types during classical conditioning of rabbit nictitating membrane response. Journal of Neurophysiology, 50, 1197-1219.

Best, P. J., \& RANCK, J. B., JR. (1982). The reliability of the relationship between hippocampal unit activity and sensory-behavioral events in the rat. Experimental Neurology, 75, 652-664.

BEST, P. J., \& THOMPSON, L. T. (1984). Hippocampal cells which have place field activity also show changes in activity during classical conditioning. Society for Neuroscience Abstracts, 10, 125.

Best, P. J., \& Thompson, L. T. (1989). Persistence, reticence, and opportunism of place-field activity in hippocampal neurons. Psychobiology, 17, 230-235.

Bostock, E., TAube, J., \& Muller, R. U. (1988). The effects of head orientation on the firing of hippocampal place cells. Society for Neuroscience Abstracts, 14, 127.

Breese, C. R., Hampson, R. E., \& Deadwyler, S. A. (1987). Direction contingent firing of hippocampal place cells. Society for Neuroscience Abstracts, 13, 608.

Breese, C. R., Hampson, R. E., \& Deadwyler, S. A. (1988). Significance of spatial location determines the firing characteristics of rat hippocampal place cells. Society for Neuroscience Abstracts, 14, 126.

Breese, C. R., Hampson, R. E., \& Deadwyler, S. A. (1989). Hippocampal place cells: Stereotypy and plasticity. Journal of Neuroscience, 9, 1097-1111.

Christian, E. P., Deadwyler, S. A. (1986). Behavioral functions and hippocampal cell types: Evidence for two nonoverlapping populations in the rat. Journal of Neurophysiology, 55, 331-348.

Eichenbaum, H., \& CoHEN, N. J. (1988). Representation in the hippocampus: What do hippocampal neurons encode? Trends in Neuroscience, 11, 244-248.

Eichenbaum, H., Kuperstein, M., Fagan, A., \& Nagode, J. (1987). Cue-sampling and goal-approach correlates of hippocampal unit ac- 
tivity in rats performing an odor discrimination task. Joumal of Neuroscience, 7, 716-732.

Foster, T. C., Castro, C. A., McNaughton, B. L. (1988). Influence of motor set on place-related hippocampal complex spike ceil activity. Sociery for Neuroscience Abstracts, 14, 396.

Foster, T. C., Christian, E. P., Hampson, R. E., Campbell, K. A., * Deadwy ler, S. A. (1987). Sequential dependencies regulate sensory evoked responses of single units in the rat hippocampus. Brain Research, 408, 86-96.

KUBIE, J. L. (1984). A driveable bundle of microwires for collecting single-unit data from freely moving rats. Physiology \& Behavior, 32. $115-118$.

Kubie, J. L., Fox, S. E., \& Muler, R. U. (1984). Variations in place firing with the state of the hippocampal EEG. Society for Neuroscience Abstracts, 10, 599.

McNaughton, B. L. (1989). Neuronal mechanisms for spatial computation and information storage. In L. Nadel, L. A. Cooper, P. Culicover, \& R. Harnish (Eds.), Neural connections and mental computations (pp. 285-350). Cambridge, MA: MIT Press.

McNaughton, B. L., Barnes, C. A., O'Keefe, J. (1983). The contributions of position, direction and velocity to single unit activity in the hippocampus of freely moving rats. Experimental Brain Research, 52, 41-49.

McNaughton, B. L., O'Keefe, J., \& Barnes, C. A. (1983). The stereotrode: A new technique for simultaneous isolation of several single units in the central nervous system from multiple unit records. Journal of Neuroscience Methods, 8, 391-397.

Morris, R. G. M. (1983). An attempt to dissociate "spatial-mapping"' and "working-memory" theories of hippocampal function. In W. Seifert (Ed.), Neurobiology of the hippocampus (pp. 405-432). London: Academic Press.

MUlLeR, R. U., \& KUBIE, J. L. (1986). Introduction of time into the study of place cells. Society for Neuroscience Abstracts, 12, 521 .

MuLler, R. U., KubIE, J. L. (1987). The effects of changes in the environment of the spatial firing of hippocampal complex-spike cells. Journal of Neuroscience, 7, 1951-1968.

Muller, R. U., Kubie, J. L., \& RANCK, J. B., JR. (1987). Spatial firing patterns of hippocampal complex-spike cells in a fixed environment. Journal of Neuroscience, 7, 1935-1950.

O'Keefe, J. (1979). A review of the hippocampal place cells. Progress in Neurobiology, 13, 419-439.
O'KeEFE, J., \& CONWAY, D. H. (1978). Hippocampal place units in the freely moving rat: Why they fire where they fire. Experimental Brain Research, 31, 573-590.

O'KeEFE, J., Dostrovsky, J. (1971). The hippocampus as a spatial map: Preliminary evidence from unit activity in the freely-moving rat. Brain Research, 34, 171-175.

OKEEFE, J., \& NADEL, L. (1978). The hippocampus as a cognitive map. Oxford: Oxford University Press.

O'Keefe, J., Speakman, A. (1987). Single unit activity in the rat hippocampus during a spatial memory task. Experimental Brain Research, 68, 1-27.

Olton, D. S., Becker, J. T., * Handelmann, G. E. (1979). Hippocampus, space, and memory. Behavioral Brain Science, 2, 313-365.

Olton, D. S., WALKer, J. A., GAGE, F. H. (1978). Hippocampal connections and spatial discrimination. Brain Research, 139, 295-308.

Parkinson, J. K., Murray, E. A., Mishkin, M. (1988). A selective mnemonic role for the hippocampus in monkeys: Memory for the location of objects. Journal of Neuroscience, 8, 4159-4167.

RANCK, J. B., JR. (1984). Head direction cells in the deep cell layer of dorsal pre-subiculum in freely moving rats. Sociery for Neuroscience Abstracts, 10, 599

Rupniak, N. M., Gaffan, D. (1987). Monkey hippocampus and learning about spatially directed movements. Joumal of Neuroscience, 7, 2331-2337

TAube, J. S., Muller, R. U., Ranck, J. B., JR. (1988). Effects of environmental manipulations on the discharge of head-direction cells in the post-subiculum. Society for Neuroscience Abstracts, 14, 126.

Thompson, L. T., Best, P. J. (1988). Hippocampal place cell to silent cell ratios in freely-behaving rats. Society for Neuroscience $A b$ stracts, 14, 126

WATANABE, T., NikI, H. (1985). Hippocampal unit activity and delayed response in the monkey. Brain Research, 325, 241-254.

Wible, C. G., Findinng, R. L., Shaptro, M., Lang, E. J., Crane, S., * Olton, D. S. (1986). Mnemonic correlates of unit activity in the hippocampus. Brain Research, 339, 97-110.

WiENER, S. I., EICHENaAUm, H. (1988). Two functional categories of hippocampal complex spike cells. Society for Neuroscience $A b$ stracts, 14, 126.

ZiPSER, D. (1985). A computational model of hippocampal place fields. Behavioral Neuroscience, 99, 1006-1017. 